\title{
Criptococosis meníngea de 1990 a 1995: hallazgos de laboratorio
}

\author{
Nelly Ordóñez, Edilma Torrado, Elizabeth Castañeda
}

\begin{abstract}
Resumen
El Laboratorio de Microbiología del Instituto Nacional de Salud ha prestado, durante los últimos 15 años, los servicios de diagnóstico, confirmación de aislamientos y determinación de las variedades y serotipos de Cryptocococcus neoformans, agente de la criptococosis. Entre 1990 y 1995, se recibieron 1.761 muestras de líquido cefalorraquídeo (LCR) y 1.068 sueros para el estudio de esta micosis; adicionalmente, se recibieron 66 aislamientos para confirmación. A partir de estas muestras, se diagnosticaron 240 casos de criptococosis en el período de seis años. Los pacientes se clasificaron en tres grupos según los resultados de la prueba serológica para VIH: $151(62,9 \%)$ VIH positivos, $43(17,9 \%)$ VIH negativos y $46(19,2 \%)$ sin el dato. EI porcentaje de pacientes VIH positivos con criptococosis se incrementó de $26,7 \%, n$ 1990 , a $80,4 \%$ en 1995. La entidad se presentó con mayor frecuencia en hombres $(80,4 \%)$ que en mujeres $(19,6 \%)$ y en el grupo de 21 a 40 años $(55 \%)$, lo que fue más evidente en el grupo VIH positivos $(67,5 \%)$. La sensibilidad y el valor predictivo negativo (VPN) del examen directo y de la aglutinación de látex en LCR y en suero presentaron valores más altos en los pacientes VIH positivos. El gran aumento en el número de casos de criptococosis es un reflejo del incremento de casos de SIDA en Colombia, epidemia que ha puesto de manifiesto el carácter oportunista de esta micosis.
\end{abstract}

\section{Summary}

Cryptococcus neoformans, the causative organism of cryptococcosis, has been diagnosed, had its isolates confirmed and its varieties and serotypes determined by the $\mathrm{Na}$ tional Health Institute's Microbiology Laboratory during the past 15 years. From 1990 to 1995, 1,761 cerebral spinal fluid (CSF) samples and 1,068 sera have been received for study; additionally, 66 isolates have been received for confirmation. Two hundred and forty cases of cryptococcosis have been determined from these samples over a six year period. Patients have been classified into three groups according to HIV serological test results, $151(62.9 \%)$ were HIV positive, $43(17.9 \%)$ HIV negative and in 46 cases $(19.2 \%)$ the data could not be obtained. The percentage of HIV positive patients with cryptococcosis increased from the $26.7 \%$ observed in 1990 to $80.4 \%$ in 1995 . The entity was presented with greater frequency in men (80.4\%) than in women (19.6\%) and in the 21 to 40 age group (55\%), this being most in evidence in the HIV positive group $(67.5 \%)$. Sera sensitivity and negative predictive values (NPV) from direct examination and latex agglutination in CSF presented higher values in HIV positive patients. The great increase in cryptococcosis cases is a reflection of the increase of AIDS cases in Colombia, an epidemic which has clearly shown this mycosis opportunist character.

Laboratorio de Microbiología, Instituto Nacional de Salud, Santa Fe de Bogotá, Colombia. 
La criptococosis es una micosis sistémica, oportunista, de amplia distribución mundial, cuyo agente etiológico es Cryptococcus neoformans, una levadura de la clase Basidiomycetes, la cual se ha clasificado en dos variedades y cuatro serotipos: $C$. neoformans var. neoformans (serotipos A y D) y $C$. neoformans var. gattii (serotipos B y C) $(1,2)$.

Esta micosis se presenta con mayor frecuencia en pacientes que tienen alguna inmunosupresión, debida tanto a condiciones de base como a tratamientos $(1,3,4)$. Es así como, con la aparición del síndrome de inmunodeficiencia adquirido (SIDA), esta micosis ha mostrado un aumento considerable en todo el mundo (3-8).

Se asume que la infección se adquiere por inhalación del hongo a partir de los microfocos en el medio ambiente, de donde es aislado frecuentemente, principalmente de suelos enriquecidos con excretas de aves (2). Aunque algunos de los pacientes con criptococosis presentan afección pulmonar aparente, generalmente es asintomática $(1,9)$. A partir del pulmón, el hongo se disemina al sistema nervioso central, debido a un tropismo especial por el mismo $(1,2)$.

La criptococosis ha sido descrita en todas las edades $(3,4,10,11)$. Antes de la aparición del SIDA, la mayoría de pacientes estaban entre los 30 y los 50 años; sin embargo, la presencia de pacientes con SIDA-criptococosis ha hecho que la presentación de la enfermedad en este rango de edad aumente aún más $(6,8,10)$.

La criptococosis y su agente etiológico han sido motivo de estudio durante los últimos 15 años en el Laboratorio de Microbiología del Instituto Nacional de Salud (INS) (11-13). Este interés particular ha hecho que se estén prestando, a nivel nacional, los servicios de diagnóstico, confirmación de aislamientos y determinación de las variedades y serotipos.

En este trabajo se revisan los casos de criptococosis diagnosticados durante el período de 1990 a 1995, y se destaca su frecuencia como enfermedad oportunista en el SIDA.

\section{Materiales y métodos}

Muestras. Entre enero de 1990 y diciembre de 1995, se recibieron 1.761 especímenes de LCR y 1.068 sueros, remitidos para investigar criptococosis. Adicionalmente, se recibieron 66 aislamientos para confirmación de género y especie. Para cada muestra, se anotó la fecha de recepción, así como el resultado del VIH y de otras condiciones de base. Se dispuso también de datos demográficos de los pacientes.

Pruebas diagnósticas. Se practicaron exámenes directos y cultivos del LCR de acuerdo con metodologías estandarizadas $(14,15)$. La identificación del hongo se realizó con base en el aspecto macroscópico y microscópico del cultivo y en el resultado de las pruebas fenotípicas, las que incluían determinación de actividad de fenol-oxidasa, ureasa, nitrato reductasa y asimilación de carbohidratos (1518).

Se realizó la determinación de la antigenemia en LCR y suero, con la prueba de aglutinación de partículas de látex (Cryptococcal antigen latex agglutination system, Meridian Diagnostics) (19).

Estudio estadístico. Se analizaron los factores de riesgo (OR) con intervalos de confianza de $95 \%(20)$.

Se estableció la sensibilidad, la especificidad y los valores predictivos positivo (VPP) y negativo (VPN) de las pruebas diagnósticas. Igualmente, se tuvo en cuenta el resultado del estudio del $\mathrm{VIH}$; el estándar de oro fue el cultivo (20).

\section{Resultados}

Los 1.544 especímenes de LCR permitieron el diagnóstico de la criptococosis en $174(11,3 \%)$ casos. De los 1.068 sueros, procesados en busca del antígeno capsular, $151(14,1 \%)$ fueron reactivos. Estos pertenecían a los 174 casos diagnosticados por métodos directos. Adicionalmente, los 66 aislamientos recibidos de otros laboratorios fueron confirmados como C. neoformans. Se contabilizaron 240 casos de criptococosis en el período de estudio. 
La positividad anual de las pruebas diagnósticas y el número de pacientes por año se encuentra en el cuadro 1 . De los 240 pacientes con criptococosis, $151(62,9 \%)$ eran VIH positivos, $43(17,9 \%)$ VIH negativos y en 46 pacientes $(19,2 \%)$ no fue posible obtener este dato (figura 1). El porcentaje de pacientes VIH positivos con criptococosis aumentó de $26,7 \%$ en 1990 a $80,4 \%$ en 1995 (figura 1).

Se registró la presencia de factores predisponentes en 11 de los 43 pacientes VIH negativus (linfoma en 5, leucemia en 3 , trauma craneoencefálico en 1, artritis reumatoidea en 1 y embarazo en 1). También se anotaron estos factores en 10 de los 56 pacientes del grupo sin dato para el VIH (linfoma en 3 , lupus eritematoso sistémico en 2, trasplante en 2, leucemia en 2 y artritis reumatoidea en 1). En uno de los 151 pacientes VIH positivos, se encontró, además, otro factor predisponente (trasplante renal).

Cuadro 1. Distribución anual de la criptococosis de acuerdo con los hallazgos en LCR y los aislamientos confirmados.

\begin{tabular}{|c|c|c|c|c|c|}
\hline \multirow[t]{3}{*}{ AÑO } & \multicolumn{3}{|c|}{ Total } & \multicolumn{2}{|c|}{$\begin{array}{c}\text { C. neoformans } \\
\text { Aislados-confirmados }\end{array}$} \\
\hline & \multirow{2}{*}{$\begin{array}{l}\text { Muestras } \\
n\end{array}$} & \multicolumn{2}{|c|}{ Pacientes } & \multirow{2}{*}{$n$} & \multirow{2}{*}{$\%$} \\
\hline & & $n$ & $\%$ & & \\
\hline 1990 & 278 & 30 & 12,8 & 49 & 17,6 \\
\hline 1991 & 235 & 35 & 16,7 & 49 & 20,9 \\
\hline & & $\begin{array}{l}32 \\
35 \\
35\end{array}$ & 11,1 & $\begin{array}{l}44 \\
55\end{array}$ & 13,5 \\
\hline 1994 & 327 & $\begin{array}{l}30 \\
57\end{array}$ & $\begin{array}{l}14,1 \\
19,4\end{array}$ & $\begin{array}{l}55 \\
77\end{array}$ & $\begin{array}{l}19,0 \\
23,6\end{array}$ \\
\hline 1995 & 372 & 51 & $\begin{array}{r}19,4 \\
15,2\end{array}$ & 71 & $\begin{array}{l}19,1 \\
19,1\end{array}$ \\
\hline TOTAL & 1827 & 240 & & 345 & \\
\hline
\end{tabular}

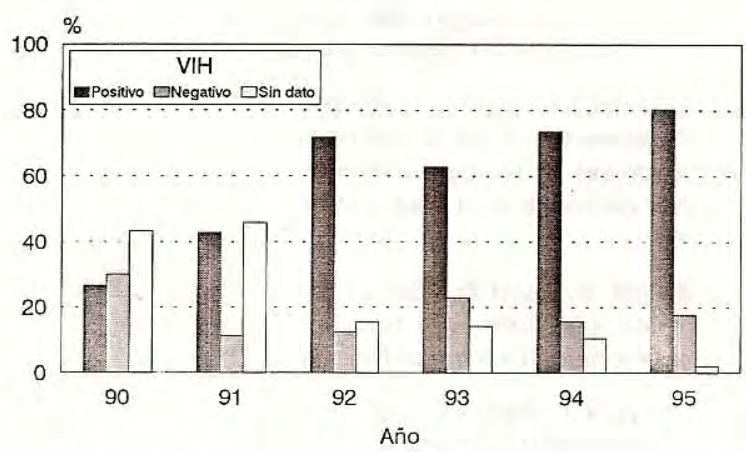

Figura 1. Distribución de los 240 casos de criptococosis según el año de diagnóstico y el resultado del VIH.
En el grupo total, la criptococosis se presentó con mayor frecuencia en hombres $(80,4 \%)$ que en mujeres (19,6\%) (cuadro 2). En el grupo de pacientes VIH positivos predominó el sexo masculino $(92,7 \%)$. No se observaron diferencias entre los grupos de pacientes $\mathrm{VIH}$ negativos y en aquéllos en quienes no se disponía del dato. En el grupo de pacientes VIH positivos, se observó un incremento de casos de criptococosis en el sexo masculino a través del tiempo; así, de 8 casos diagnosticados en 1990 se pasó a 36 en 1995. Las 11 mujeres fueron diagnosticadas entre 1994 y 1995.

En todos los grupos analizados, se observó una mayor incidencia de criptococosis en pacientes con edades entre 21 y 40 años (55\%). Tal incidencia fue más alta en el grupo de pacientes VIH positivo (figura 2). Se destacó también la presencia de la micosis en un niño VIH positivo $(0,7 \%)$.

Cuadro 2. Distribución de los 240 pacientes con criptococosis según año, resultado del VIH y sexo.

\begin{tabular}{|l|c|l|l|l|}
\hline \multirow{2}{*}{ AÑO } & \multicolumn{4}{|c|}{$\begin{array}{c}\text { Resultado VIH y relación entre sexos } \\
\text { Masculino : Femenino }\end{array}$} \\
\cline { 2 - 5 } & Positivo & Negativo & Sin dato & Total \\
\hline 1990 & $8: 0$ & $5: 4$ & $10: 3$ & $23: 7$ \\
1991 & $15: 0$ & $2: 2$ & $9: 7$ & $26: 9$ \\
1992 & $23: 0$ & $3: 1$ & $2: 3$ & $28: 4$ \\
1993 & $22: 0$ & $5: 3$ & $1: 4$ & $28: 7$ \\
1994 & $36: 6$ & $5: 4$ & $4: 2$ & $45: 12$ \\
1995 & $36: 5$ & $6: 3$ & $1: 0$ & $43: 8$ \\
\hline Relación & $13: 1$ & $1.5: 1$ & $1.4: 1$ & $4: 1$ \\
\hline
\end{tabular}

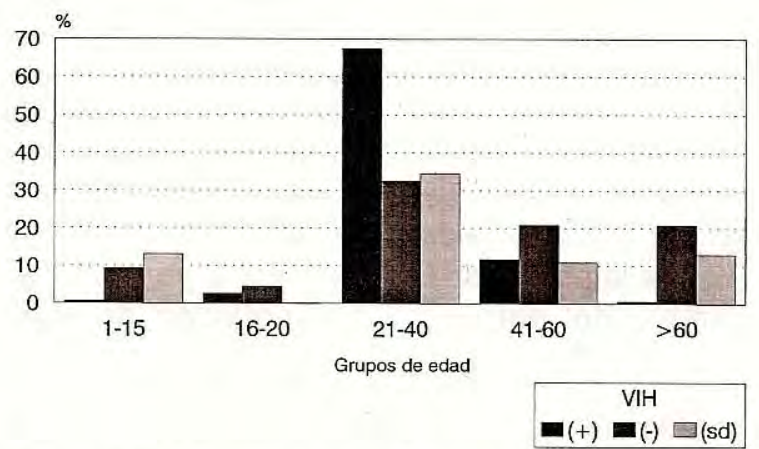

Figura 2. Distribución de los 240 casos de criptococosis según grupos de edad y resultado del VIH de los pacientes. 
En los estudios de riesgos relativos (OR) fue significativo ser $\mathrm{VIH}$ positivo $(\mathrm{OR}=11,22$, IC $95 \%=8,17$ a 15,4$)$.

En el cuadro 3 se ilustra la sensibilidad y el valor predictivo negativo (VPN) del examen directo y de la prueba de aglutinación con partículas de látex. La especificidad y el valor predictivo positivo (VPP) para estas pruebas fue del $100 \%$.

\section{Discusión}

El aumento en el número de muestras remitidas al Laboratorio del Instituto Nacional de Salud entre 1990 y 1995, señala no sólo un mejor conocimiento del clínico sobre la función de apoyo del laboratorio en el diagnóstico de la criptococosis, sino también sobre la mayor importancia de esta micosis en la población VIH positiva (4).

El aumento en la frecuencia de casos de criptococosis por año, que pasó de 12,8\% observado en 1990 a $19,4 \%$ en 1994 , es debido, como en el resto del mundo, al aumento de pacientes VIH positivos. En esta serie, los pacientes con esta infección oportunista aumentaron de $26,7 \%$, en 1990 , a $80,4 \%$ en $1995(6-8)$.

El resultado de $\mathrm{VIH}$, el sexo masculino y la edad, constituyeron los principales factores de riesgo para la aparición de esta micosis. Estos hallazgos confirman lo ya informado en la literatura $(3-8,21)$.

El predominio marcado de la criptococosis en hombres jóvenes, con edades entre 21 y 40 años, es un reflejo de la población afectada por el SIDA en nuestro país, lo cual es similar a lo informado en otros países latinoamericanos como Argentina $(8,22)$. No obstante, llama la atención la aparición reciente de un número de casos en mujeres y en un niño, lo que está de acuerdo con la extensión de la epidemia de SIDA a la población heteroxesual y, por ende, al cambio del patrón de presentación de este síndrome (23).

Al igual que en los casos estudiadas por Clarck y Bava $(7,8)$, en esta serie se observó que el número de casos de criptococosis en pacientes $\mathrm{VIH}$ positivos, se incrementaba mientras que el
Cuadro 3. Sensibilidad y valor predictivo negativo de las pruebas diagnósticas en los pacientes $\mathrm{VIH}(+)$ y $\mathrm{VIH}(-)$

\begin{tabular}{||l|c|c|c|l||}
\hline \multirow{2}{*}{$\begin{array}{c}\text { Pruebas } \\
\text { estadisticas }\end{array}$} & \multirow{2}{*}{$\begin{array}{c}\text { Resultado } \\
\text { VIH }\end{array}$} & \multicolumn{3}{|c|}{ Pruebas diagnósticas } \\
\cline { 3 - 5 } & & $\begin{array}{c}\text { Examen } \\
\text { directo }\end{array}$ & \multicolumn{2}{|c|}{ Látex } \\
\cline { 3 - 5 } & & & LCR & Suero \\
\hline Sensibilidad & $(+)$ & 89,0 & 97,6 & 93,9 \\
& $(-)$ & 86,4 & 95,7 & 66,7 \\
Valor predictivo & $(+)$ & 94,6 & 98,8 & 96,9 \\
negativo (VPN) & $(-)$ & 90,6 & 95,5 & 84,8 \\
\hline
\end{tabular}

de pacientes VIH negativos permanecía estable. Esta infección oportunista se había observado asociada al SIDA en el $10,9 \%$ de los pacientes estudiados en Medellín en 1993 (22).

Las variaciones en la sensibilidad y VPN que presentaron las pruebas diagnósticas en relación con el grupo de pacientes $\mathrm{VIH}$, pueden explicarse porque las técnicas diagnósticas empleadas detectan antígeno, el cual está presente en más alta concentración en los pacientes VIH positivos que en los VIH negativos, tanto en las muestras de LCR como de suero $(5,24,25)$.

Se resalta la alta incidencia que, en general, presenta la criptococosis en Colombia, destacándose la afección en pacientes del sexo femenino y en niños VIH positivos, la cual viene ocurriendo recientemente.

\section{Agradecimientos}

A la doctora Angela Restrepo.

\section{Referencias}

1. Treseler CG, Sugar AM. Fungal meningitis. Infect Dis Clin North Am 1990;4:789-808.

2. Kwon-Chung KJ, Kozel TR, Edman JC, Polacheck I, Ellis D, Shinoda T, Dromer F. Recent advances in biology and immunology of Cryptococcus neoformans. J Med Vet Mycol 1992;30 sup I:133 -42 .

3. Speed B, Dunt D. Clinical and host differences between infections with two varieties of Cryptococcus neoformans. Clin Infect Dis 1995;21:28-34.

4. Vergara I, Saavedra M, Saravia J, González G, Lorenzana P, Acosta C, Cuervo S, Pardo G, Toro G. Criptococosis del sistema nervioso central. Estudio de 32 casos, 1975-1991. Acta Med Colomb 1993;18:199-210. 
5. Dupont B, Graybill JR, Armstrong D, Laroche R, Touze JE, Wheat LJ. Fungal infections in AIDS patients. J Med Vet Mycol 1992;30 sup I:19-28.

6. Guerrero A, Navas E, Redonde E, Hermida J, Sánchez A. Criptococosis. Diagnóstico clínico y experiencia hospitalaria en la era del SIDA. Rev Iberoamericana de Micología 1994;11:77-80.

7. Clarck RA, Greer D, Atkinson W, Valainis GT, Hyslop N. Spectrum of Cryptococcus neoformans infection in 68 patients infected with human immunodeficiency virus. Rev Infect Dis 1990;12:768-7.

8. Bava A, Negroni R. Características epidemiológicas de 105 casos de criptococosis diagnosticados en la República Argentina entre 1981-1990. Rev Inst Med Trop Sao Paulo 1992;34:335-40.

9. Driver JA, Saunders CA, Heinze-Lacey B, Sugar AM. Cryptococcal pneumonia in AIDS: Is cryptococcal meningitis preceded by clinically recognizable pneumonia? J Acq Imm Def Syndrom and Human Retrovirol 1995;9:168-71.

10 Kwon-Chung KJ, Bennett JE. Cryptococcosis. In: Kwon-Chung KJ, Bennett JE, editors. Medical mycology. Philadelphia, 1992:397-446.

11. Ordóñez N, Castillo J, Moreno G. Criptococosis: diagnóstico por el laboratorio. Biomédica 1987;7:37-41.

12. Ordóñez N, Castañeda E. Criptococosis. Estudio de catorce casos con diagnóstico comprobado por el laboratorio. Biomédica 1981;1:87-93.

13. Ordóñez N, Castañeda E. Serotipificación de aislamientos clínicos y del medio ambiente de Cryptococcus neoformans en Colombia. Biomédica 1994;14:131-9.

14. McGinnis M. Media and reagents. In: Laboratory handbook of medical mycology. New York: Academic Press, 1980:529.
15. Kreger Van Rij. The yeasts: a taxonomic study. Amsterdam: Elsevier Science Publishers, 1984.

16. Seeliger $\mathbf{H}$. Use of urease test for the screening and identification of Cryptococci. J Bacteriol 1956;72:127 -31 .

17. Hopkins JM, Land GA. Rapid method for determining nitrate utilization by yeasts. J Clin Microbiol 1977; 5:407-8.

18. Adams ED, Cooper BH. Evaluation of a modified Wickerham medium for identifying medically important yeasts. Amer J Med Technol 1974;40:377-8.

19. Palmer D, Kaufman L, Kaplan W, Cavallaro J. Serodiagnosis of mycotic diseases. Springfield: Charles C Thomas, 1977:76-106.

20. Fleiss J. Statistical methods for rates and proportions. New York: John Wiley \& Sons, 1981:1-18.

21. Dupont B, Denning D, Marriott D, Sugar A, Viviani M, Sirisanthana T. Mycosis in AIDS patients. J Med Vet Mycol 1994;32:65-77.

22. Velásquez G, Betancur J, Estrada S, Nagles J, Orozco B, Ospina S, et al. Infecciones observadas en 193 pacientes con SIDA. Estudio multicéntrico en la ciudad de Medellín 1987-1992. Acta Med Colomb 1993; 18:56-65.

23. Martínez M, Rodríguez R. Infección por VIH/SIDA en Colombia. Boletín epidemiológico Instituto Nacional de Salud 1994;2:33.

24. Shahab T. Significance of cryptococcal antigen titers in patients with AIDS. Southern Med J 1991;84:14078.

25. Laroche R, Dupont B, Touze JE, Taelman H, Bogaerts J, et al. Cryptococcal meningitis associated with acquired immunodeficiency syndrome (AIDS) in African patients: treatment with $f$ luconazole. J Med Vet Mycol 1992;30:71-8. 